Didáctica. Lengua y literatura

ISSN: 1130-0531

http://dx.doi.org/10.5209/DIDA.61959

\title{
Autoevaluación y evaluación negociada entre docente y alumnos en la clase de pronunciación inglesa: un estudio con estudiantes argentinos
}

\author{
Pedro Luis Luchini ${ }^{1}$; Gabriela Mariel Ferreiro²
}

Recibido: 12 de enero de 2015 / Aceptado: 15 de febrero de 2015

Resumen. Establecer el impacto de la enseñanza en el aprendizaje presupone la utilización de diversos procedimientos y técnicas evaluativas. La evaluación conlleva la recolección de información sobre la calidad y la cantidad del cambio experimentado por un alumno o un grupo de alumnos. La calificación, por su parte, es el acto de juzgar el mérito, el valor o la correspondencia de un determinado rendimiento producido por los alumnos. Enseñanza, aprendizaje, evaluación y calificación son conceptos que están estrechamente relacionados entre sí e interactúan de manera habitual en la práctica docente. Este proyecto tiene como propósito determinar los efectos de la autoevaluación y la evaluación negociada entre docente y alumnos en una clase de pronunciación del inglés. Según lo señalan los resultados de estudios previos (Luchini, 2003; 2006), las tareas que integran un componente de autoevaluación y negociación estimulan la toma de conciencia lingüística y reactivan la motivación de los alumnos para mejorar su pronunciación en LE (Lengua Extranjera). Participaron en el presente trabajo de investigación 17 estudiantes de un curso que se dicta en el segundo año del Profesorado de Inglés universitario en la ciudad de Mar del Plata, Argentina. Se trata de un curso de pronunciación, con foco en la prosodia inglesa. Los alumnos grabaron sus producciones orales y las transcribieron. Posteriormente, utilizando una rúbrica, evaluaron y calificaron sus producciones. Sus calificaciones fueron posteriormente comparadas con las asignadas por su docente. Los resultados revelaron que las tareas de evaluación estimularon en los alumnos una mayor concienciación lingüística, autonomía y motivación para continuar aprendiendo.

Palabras clave: Pronunciación en LE; autoevaluación; evaluación negociada; concientización lingüística; autonomía; motivación.

\section{[en] Self-assessment and Negotiated Evaluation Among Teacher and Students in the Pronunciation Class: A Study with Argentinean Learners}

\begin{abstract}
To determine the impact of teaching on learning calls for a number of precise evaluative procedures. Evaluation implies collecting data about the quality and quantity of change experimented by students. Assessment, on the other hand, is associated with judgment and measurement of students' performance. Teaching, learning, evaluation and assessment are closely related and function jointly in practice. The aim of this study is to determine the effects and implications of self-evaluation and negotiated evaluation among teacher and students in the L2 (second/foreign language) pronunciation class. Based on previous studies (Luchini, 2003; 2006), tasks that integrate self-evaluation and negotiation stimulate consciousness raising and enhance students' motivation to improve their L2 pronunciation. Seventeen trainees from Argentina (Spanish-L1 speakers) participated in this experiment. At the time of data collection, they were enrolled in an English pronunciation course with a strong focus on prosody

$1 \quad$ Universidad Nacional de Mar del Plata. Facultad de Humanidades, Dpto. de Lenguas Modernas (Argentina) E-mail: luchinipedroluis@gmail.com

2 Universidad Nacional de Mar del Plata. Facultad de Humanidades, Dpto. de Lenguas Modernas (Argentina)

E-mail: louis.assierandrieu@sciencespo.fr
\end{abstract}


in a state-owned university in Mar del Plata, Argentina. The students recorded a speech sample a later transcribed it. They analyzed their production, and based on a speaking rubric graded their work. Their grades were later compared with those coming from their teacher who also listened to and evaluated their recordings. Results revealed that these evaluative tasks contributed to develop the students' phonological consciousness and their autonomy and motivation to keep on learning.

Keywords: L2 pronunciation; self-assessment; negotiated evaluation; consciousness raising; learner autonomy; motivation.

\section{[fr] Autoévaluation et évaluation négociées entre l'enseignant et les étudiants dans le cours de prononciation en anglais: une étude avec des étudiants argentins}

Résumé. L'impact de l'enseignement sur l'apprentissage suppose l'utilisation de diverses procédures et techniques d'évaluation. L'évaluation implique la collecte d'informations sur la qualité et la quantité du changement vécu par un étudiant ou un groupe d'étudiants. La qualification, en revanche, est l'acte de juger du mérite, de la valeur ou de la correspondance d'une certaine performance produite par les étudiants. L'enseignement, l'apprentissage, l'évaluation et la qualification sont des concepts étroitement liés entre eux et interagissant de manière régulière dans la pratique de l'enseignement. Le but de ce projet est de déterminer les effets de l'auto-évaluation et de l'évaluation négociée entre l'enseignant et les élèves dans un cours de prononciation en anglais. Comme l'indiquent les résultats d'études antérieures (Luchini, 2003, 2006), les tâches qui constituent un composant d'autoévaluation et de négociation stimulent la prise de conscience linguistique et réactivent la motivation des étudiants à améliorer leur prononciation en LE (langue étrangère). Ont participé au travail de recherche actuel 17 étudiants d'un cours qui est enseigné en deuxième année de la faculté de l'université anglaise dans la ville de Mar del Plata, en Argentine. C'est un cours de prononciation, axé sur la prosodie anglaise. Les étudiants ont enregistré leurs productions orales et les ont transcrites. Plus tard, en utilisant une rubrique, ils ont évalué et évalué leurs productions. Ses notes ont ensuite été comparées à celles de son professeur. Les résultats ont révélé que les tâches d'évaluation stimulaient chez les élèves une plus grande conscience linguistique, une plus grande autonomie et une motivation accrue pour continuer à apprendre.

Mots Clés: Prononciation en LE; auto-évaluation; évaluation négociée; prise de conscience linguistique; autonomie; motivation.

Sumario. 1. Introducción. 2. Marco teórico. 3. Preguntas conducentes a la investigación. 4. Método. 4.1. Contexto y participantes. 4.2. Instrumentos. 4.3. Procedimiento. 5. Resultados. 5.1. Las calificaciones. 5.2. Autoinformes evaluativos de los alumnos. 6. Discusión. 7. Conclusión. 8. Limitaciones del estudio. 9. Referencias bibliográficas. 10. Apéndices.

Cómo citar: Luchini, P. L.; Ferreiro, G. M. (2018) Autoevaluación y evaluación negociada entre docente y alumnos en la clase de pronunciación inglesa: un estudio con estudiantes argentinos, Didáctica. Lengua y Literatura, 30, 137-156.

\section{Introducción}

Durante mucho tiempo la enseñanza de la pronunciación del inglés como LE (Lengua Extranjera) fue relegada de la agenda de la Lingüística Aplicada. Sin embargo, con el advenimiento de la globalización y la necesidad de una lengua franca para el logro de una comunicación internacional efectiva, el estudio de la pronunciación ha recuperado su vigencia en la enseñanza de LEs. Así es como Jenkins (2000), al 
reflexionar acerca del rol de la pronunciación en la interacción verbal, sostiene que la incompetencia en esta habilidad suele dar lugar a la ininteligibilidad. Por otra parte, autores como Galaczi, Post, Li, Barker y Schmidt (2015) se expresan al respecto y advierten que pese al resurgimiento del interés en la pronunciación, esta aún tiene asignado un lugar secundario en algunos modelos teóricos de adquisición de LEs, en escalas y rúbricas de evaluación de la oralidad y en algunas prácticas evaluativas y contextos áulicos, donde los protagonistas son los docentes, evaluadores y alumnos.

Con el propósito de determinar el impacto de la enseñanza en el aprendizaje, en este caso de la pronunciación en LE, resulta necesario recurrir al uso de procedimientos evaluativos. En el contexto de la enseñanza, evaluar implica recabar datos sobre la calidad y la cantidad del cambio experimentado por los alumnos en su proceso de aprendizaje. El presente trabajo tiene como objetivo analizar el efecto de la implementación de dos técnicas de evaluación en el proceso de adquisición de la pronunciación del inglés como LE: la autoevaluación y la evaluación negociada. Participaron en este proyecto un grupo de 17 alumnos universitarios argentinos cuya lengua madre es el español.

A continuación, se presenta el marco teórico donde se amplían y delimitan los conceptos y nociones que se utilizarán a lo largo del estudio. También, se describen los participantes y el contexto experimental y se detallan los instrumentos utilizados para la recolección de datos. Posteriormente, se analizan e interpretan los hallazgos para luego brindar algunas conclusiones generales. Por último, se ofrecen algunas recomendaciones y se presentan propuestas de investigación para continuar explorando el área.

\section{Marco teórico}

La enseñanza de la pronunciación del inglés y su estudio dentro de la agenda de la Lingüística Aplicada son temas que han recobrado notable interés en la actualidad entre docentes e investigadores. Este resurgimiento de la pronunciación impacta en algún punto en la enseñanza de la oralidad y la investigación. Según Murphy y Baker (2015), la interacción verbal debe considerar cómo el inglés se pronuncia para dar cuenta de lo que sucede en cualquier otro ámbito lingüístico del discurso oral (análisis del discurso, pragmática, variación lingüística, análisis socioculturales de la lengua, adquisición de la lectura, el inglés como lengua franca, entre otros), porque la pronunciación es reconocida como una variable muy importante, y como tal no debe ser ignorada.

Pese a lo previamente expuesto, la pronunciación parecería continuar teniendo un rol secundario en lo que respecta a las descripciones de varios modelos teóricos de adquisición de LE, en los diseños y elaboración de rúbricas y escalas para la evaluación de la oralidad y en la evaluación del habla en contextos áulicos. En relación con los modelos teóricos, la pronunciación, junto con la pragmática, continúan siendo marginalmente tratadas, en comparación con otros aspectos lingüísticos como el vocabulario y la gramática (Isaacs, 2014). Este rol marginal asignado a la pronunciación también se observa en el diseño de escalas y rúbricas de evaluación del habla, en las que la pronunciación es incongruentemente tratada y, en algunos casos, hasta ignorada por completo. Por ejemplo, el ACTFL Proficiency Guidelines for 
Speaking (ACTFL, 2012) no incluye descriptores de la pronunciación en ninguno de sus niveles de competencia lingüística. Del mismo modo, el Common European Framework of Reference for Languages (CEFR) omite la pronunciación como un criterio de evaluación en su escala evaluativa (Council of Europe, 2001). Atribuyen esta decisión, en parte, a la dificultad en distinguir los diferentes niveles de habilidad de pronunciación, de la misma forma que sucede con otras habilidades lingüísticas y a lo difícil que resulta interpretar los descriptores de pronunciación para cada idioma (North \& Hughes, 2003).

Este desafío frente al constructo de la pronunciación de LE también se pone de manifiesto entre algunos docentes y examinadores. Numerosos estudios han dado cuenta de que al examinar la oralidad, los evaluadores señalan que la pronunciación resulta ser la habilidad lingüística más desafiante de todas, y que sienten mayor confianza cuando hacen evaluaciones globales del habla, como por ejemplo de inteligibilidad, que cuando deben realizar evaluaciones a nivel micro-fonético-fonológico, tanto de aspectos segmentales como suprasegmentales (Brown \& Taylor, 2006; Isaacs, Trofimovich, Gu \& Chereau, 2015; Yates, Zielinski \& Pryor, 2011). Asimismo, muchos docentes no cuentan con el conocimiento y entrenamiento necesarios para enseñar pronunciación efectiva y eficazmente (Levis, 2005).

Por medio de este estudio, se intentará brindar algunas respuestas a estas necesidades e inquietudes. En especial, se abordará el tema del impacto que pueden alcanzar la evaluación y la autoevaluación negociada entre docente y alumnos en la clase de pronunciación del inglés como LE. En este punto, resulta oportuno definir los términos "pronunciación", "evaluación", "autoevaluación", debido a que aparecerán con frecuencia a lo largo del trabajo. La "evaluación negociada" también será foco de análisis, en este caso, en lo que refiere a la asignación de un índice de calificación no solo por parte del docente sino por parte de los alumnos sobre un mismo trabajo de producción oral.

En el contexto de este trabajo de investigación, cuando se habla de "pronunciación" se hace referencia a los sonidos individuales: vocales y consonantes, comúnmente referidos como segmentos. Por otro lado, los rasgos prosódicos, también conocidos como aspectos suprasegmentales, involucran una serie de rasgos un tanto más abarcadores que los sonidos y que operan por sobre encima de ellos. Se trata del acento, el ritmo y la entonación (Isaacs \& Trofimovich, 2017).

El presente estudio no supone la evaluación como un instrumento de certificación de aprendizajes o de supervisión docente, sino como un proceso de toma de conciencia de los aprendizajes adquiridos y de las dificultades que se presentan en la adquisición, la comprensión o en la transferencia de conocimientos (Bachman, 2004). En efecto, la evaluación es indagada desde una perspectiva didáctica, como una fuente generadora de información (Litwin, 1998); es decir, para rever el aprendizaje, reflexionar acerca de la enseñanza y explorar las relaciones y las implicancias de estos dos procesos con el objetivo de mejorar las prácticas docentes.

Como fuera señalado anteriormente, la evaluación implica reunir información sobre la calidad y la cantidad del cambio experimentado por los alumnos en torno a su proceso de aprendizaje. Y en este punto es necesario definir y aclarar el término "calificación" de la manera en que será abordado en este estudio. Calificación es el acto de medir el mérito, el valor o la correspondencia de un determinado rendimiento realizado por parte de los alumnos. Cabe aclarar que se puede evaluar sin tener que calificar, pero no se puede calificar sin evaluar. Y la calidad de la 
evaluación determina, en gran medida, la calidad de las calificaciones (Johnson \& Johnson, 2004).

En este trabajo, se ha empleado la técnica de la autoevaluación y evaluación negociada con el propósito de determinar la naturaleza de su impacto y para estimular en los alumnos la conciencia lingüística, en particular la fonético-fonológica, al escuchar y valorar sus propias producciones orales en LE, monitorear sus logros y sugerir áreas que necesiten ser modificadas para lograr un acento cercano al de los hablantes nativos de inglés. En este contexto entendemos la concientización lingüística como el fenómeno por medio de la cual el aprendiz debe atender y reconocer las formas lingüísticas del input al que está expuesto si se espera que estas formas luego se transformen en un constructo del aprendizaje. Intervienen en este proceso un número de mecanismos y subsistemas: alerta, orientación, detección por medio de la atención selectiva, facilitación e inhibición (Schmidt 2001, 2010; Tomlin \& Villa, 1994).

Se apunta a este objetivo porque estos alumnos, futuros profesionales de la lengua (profesores de inglés), deberán ser capaces de comunicarse efectivamente con hablantes nativos de inglés, con otros hablantes no nativos de inglés de diferentes nacionalidades y con sus propios alumnos (de la nacionalidad donde deban enseñar). Además, como futuros profesionales de la lengua deberán interactuar y mostrarse como buenos modelos y ejemplos de desarrollo lingüístico en LE.

En 2003 y 2006, uno de los autores (Luchini) realizó dos trabajos de investigación en los que analizó el impacto de la autoevaluación y evaluación negociada, respectivamente. En el primer estudio, 23 alumnos de inglés de la carrera de Traductor Público de una universidad privada en Mar del Plata, Argentina, grabaron sus producciones orales, las escucharon, las transcribieron y, finalmente, se asignaron una calificación y compararon sus resultados con los obtenidos por su docente. Los resultados revelaron que la tarea de evaluación negociada tuvo un impacto positivo sobre sus procesos de aprendizaje de la pronunciación en LE. También, resaltaron este tipo de tareas, porque por medio de ellas desarrollaron mayor concientización fonológica, autonomía y motivación para continuar aprendiendo. En el segundo estudio, el mismo docente-investigador analizó el efecto de la autoevaluación con 268 alumnos chinos de la Universidad Normal de Shanghai, China. Los hallazgos provenientes de autoinformes y cuestionarios indicaron que la tarea de autoevaluación contribuyó a estimular en los alumnos la concientización lingüística y a promover el monitoreo de sus producciones, factores que, según los participantes, contribuyeron a desarrollar su pronunciación en inglés.

Hay varias formas y técnicas de llevar a cabo la autoevaluación. Estas formas son dúctiles a cada contexto y suelen ser seleccionadas según su propósito (Boud, 2003; Fernández López, 2017). La autoevaluación puede adoptar distintos formatos, tales como lo son las pruebas (orales/escritas), los diarios de aprendizaje (en inglés, diaries/journals), las hojas de plan semanal/mensual, los informes auto evaluativos, las grabaciones y las transcripciones (audio/audiovisuales), los portfolios, los debates, los grupos focales, las planillas de seguimiento del estudiante, las escalas, grillas y rúbricas para la corrección, los cuestionarios y las entrevistas (Boud, 2003; Coombe y Canning, 2002; Oscarson, 1989; Fernández López, 2017).

Los nuevos enfoques de enseñanza de LEs, centrados en el desarrollo de la comunicación (Altamimi, 2015; Baker-Smemoe \& Haslam, 2012; Baran-Lucarz, 2012; Taylor, 1991; Morley, 1991, 1994; Rose, 2012; Moyer, 2014, 2015), consideran al 
alumno como un protagonista principal en el proceso de aprendizaje (Farrell \& Jacobs, 2010). En estos enfoques comunicativos se visualiza un desplazamiento desde una mirada grupal hacia un enfoque creciente en la unicidad del individuo, teniendo en cuenta las diferencias individuales, estilos y estrategias de aprendizaje y la motivación. Se centran en la participación de tanto los alumnos como los docentes, con un gran énfasis en el desarrollo de la conciencia del habla y la metacognición, en especial las estrategias de auto-monitoreo (Murphy, 1998). Los alumnos asumen la responsabilidad de su resultado académico, porque el docente actúa como facilitador del desarrollo de estrategias de auto-corrección y evaluación en sus alumnos, y los motiva a tomar conciencia de su propio potencial como partícipes activos en su proceso de aprendizaje. En efecto, el docente se desempeña como ayudante y asistente de sus esfuerzos. Este nuevo rumbo en la enseñanza de LE también ha tenido su impacto en la enseñanza de la pronunciación del inglés como LE, porque a partir de estos nuevos enfoques esta habilidad es concebida como una parte integral de la comunicación y no como un área subsidiaria aislada. Así entonces, en la clase de pronunciación, los alumnos cumplen la función de participantes activos, porque se los invita a participar en la toma de decisiones y a compartir responsabilidades en su proceso de aprendizaje.

Al desarrollar un método independiente de auto-monitoreo y asumir un rol de mayor responsabilidad frente a su propio aprendizaje, es de esperar que los alumnos se sientan más cómodos y seguros al utilizar la LE en el aula. No sería ilógico, entonces, decir que de esta manera se incentiva su motivación (Dörnyei, 2001, 2012; Dörnyei, Henry \& Muir, 2016; Ordorica, 2010) por aprender. Se entiende que la motivación juega un rol importante en el proceso de aprendizaje, porque actúa como un filtro afectivo que permite o no que el input alcance la parte del cerebro donde el aparato de la adquisición lingüística está localizado (Krashen, 1985). Por otro lado, Gardner (1988) sostiene que un alumno motivado aprende mejor que uno no motivado, porque el motivado presta mayor atención y atiende más selectivamente a los aspectos morfosintácticos y fonético-fonológicos de la lengua meta, sin detenerse en el contenido. A su vez, el prestar mayor atención genera mayores instancias de advertencia hacia la lengua (en inglés, noticing), y los alumnos motivados suelen ser más perseverantes y dedicados que los que no lo son, por lo tanto, logran un entendimiento más elevado (en inglés, understanding) acerca de la importancia del lenguaje que advierten, manifestándose en niveles más altos de reconocimiento (en inglés, awareness) y aprendizaje en general. El esfuerzo combinado con la perseverancia y la atención pareciera ser la combinación ideal que facilita la adquisición lingüística (Schmidt, 2010).

Una técnica que también permite a los alumnos tomar conciencia acerca de su propio proceso de aprendizaje es la evaluación negociada. Boud y Kilty (2003) definen la evaluación negociada como el proceso sistemático y recursivo mediante el cual se determina el grado en que se están logrando los objetivos propuestos. Por su parte, Font Ribas (2003) sugiere que tanto los criterios de evaluación como los resultados de esa misma evaluación pueden ser objeto de negociación entre docentes y alumnos. Este proceso evaluativo implica descripciones numéricas y juicios de valor basados en la interpretación de las descripciones y pueden participan todos los intervinientes en el proceso de aprendizaje, en especial los alumnos y los docentes (Celman, 1998).

Las evaluaciones son efectivas cuando los docentes y los alumnos, con la información obtenida, relacionan datos, formulan posibles hipótesis y pronuncian juicios 
fundados que permiten una mejor comprensión de lo que sucede, cómo sucede y por qué (Celman, 1998). Por medio de la evaluación negociada los alumnos pueden tener un mayor control de su aprendizaje al compartir el poder con el docente (Dickinson, 1995; Wong, 2015), y al recibir instrucciones específicas y guías de participación con metas precisas que los ayuden a desarrollar una conciencia de estudio, una capacidad de monitoreo del habla y estrategias de modificación discursiva que puedan emplear tanto en el aula como fuera de ella (Murphy, 1998; Taylor, 1991).

En la próxima sección se describirán las preguntas conducentes a la investigación, el contexto, los participantes, los instrumentos para la recolección de datos y los procedimientos empleados.

\section{Preguntas conducentes a la investigación}

Una enseñanza de calidad presupone la aplicación de procedimientos evaluativos. Tal como fuera mencionado anteriormente, partimos de la hipótesis en la que se plantea que las técnicas de autoevaluación y de evaluación negociada contribuyen al desarrollo de la pronunciación en LE en tanto que promueven la concientización lingüística, favorecen el desarrollo de la autonomía en el aprendizaje y generan e incentivan en los alumnos la motivación por continuar aprendiendo. Con el objetivo de corroborar esta hipótesis y delimitar el objetivo del estudio, se plantean las siguientes preguntas:

1. ¿En qué medida la autoevaluación y la evaluación negociada contribuyen a despertar y estimular la concientización lingüística en los alumnos acerca del rol que cumple la pronunciación en el desarrollo del discurso oral en LE?

2. ¿En qué medida la autoevaluación y la evaluación negociada favorecen el desarrollo de la autonomía en el aprendizaje?

3. ¿En qué medida la autoevaluación y la evaluación negociada incentivan la motivación por mejorar el desarrollo de la pronunciación en LE?

\section{Método}

\subsection{Contexto y participantes}

Este estudio se desarrolló en el contexto de la asignatura Discurso Oral II (DOII). DOII es un curso de pronunciación inglesa, con foco en la prosodia y la lengua en uso. El curso tiene una duración de cuatro meses y se dicta en el segundo año del Profesorado de Inglés de la Universidad Nacional de Mar del Plata, Argentina. Los alumnos acceden a este curso luego de aprobar Fonética y Fonología I y II, dos asignaturas que se dictan en el primer año de la carrera, en las que se estudia la descripción y naturaleza de los sonidos del inglés.

El objetivo principal de DOII es lograr que los alumnos mejoren su pronunciación en LE. Mediante la concreción de tareas comunicativas, con foco en el desarrollo de la fonología suprasegmental, y seguidas de períodos cortos de análisis metalingüístico y reflexivo, los alumnos son estimulados a tomar conciencia fonológica 
y a desarrollar una actitud activa e independiente de cara al aprendizaje. El nivel de competencia lingüística en inglés de los 17 alumnos que participaron en este estudio fue equivalente al C1, según lo señala el estándar internacional del Marco Común de Referencia (MCER). El rango de edad de los participantes fue de 19-27 años, con una media de 19.88. El grupo estuvo conformado mayormente por participantes femeninos, excepto por uno de ellos. El docente a cargo del curso y de implementar estas tareas cumplió los roles de asistente, asesor y evaluador del proyecto.

\subsection{Instrumentos}

Los instrumentos utilizados para la recolección de datos fueron las hojas de desempeño (Apéndice A) donde el docente volcó su devolución y comentarios acerca del desempeño de los alumnos y sus informes auto-evaluativos (Apéndice B). Las muestras de habla de los alumnos consistieron en una instancia de grabación de habla espontánea, con una duración de 1 minuto aproximadamente, en la que se les solicitó que comparen y contrasten dos fotos. Los alumnos completaron sus grabaciones, las transcribieron ortográficamente, las analizaron y finalmente se asignaron una calificación, guiados por la rúbrica de calificaciones previamente diseñada para evaluar el habla oral (ver Cuadro 1). Posteriormente, escribieron sus opiniones y percepciones con respecto a la implementación de la tarea evaluativa y a su impacto en el desarrollo del discurso oral en LE, siempre focalizados en el desarrollo de la pronunciación.

\subsection{Procedimiento}

En este curso, los alumnos deben aprobar 3 de 4 trabajos prácticos en clase y 2 exámenes parciales. De los 4 trabajos prácticos, el primero, que se realizó durante la tercera semana de clases, consistió en una tarea que incluyó la técnica de autoevaluación y evaluación negociada entre el docente y los alumnos. Estas tareas tuvieron un doble propósito. Por un lado, lograr que los alumnos desarrollaran una mayor conciencia fonológica de aquellas formas que necesitaban modificar para lograr adecuación fonológica y poder comunicarse efectivamente (Jenkins, 2000; Munro \& Derwing, 1995, 1998, 2015; Singhanuwananon, 2016), y por el otro, hacer que los alumnos compartieran con el docente la responsabilidad de evaluar sus producciones y reflexionar, en conjunto, acerca del proceso de aprendizaje y de enseñanza de la pronunciación en LE.

Estas tareas evaluativas fueron llevadas a cabo en el laboratorio de idiomas de la universidad donde, a cada alumno individualmente se le pidió que observe dos fotos (1 minuto) para que, posteriormente, las compare y contraste. Los estímulos visuales, como el que se utilizó en esta oportunidad, resultan ser herramientas efectivas para generar en los alumnos un tópico acerca del cual puedan conversar espontáneamente, sin haberlos provisto previamente de palabras o frases que utilizadas (Underhill, 1987). A pesar de que el tópico de conversación se encuentra, de alguna forma, limitado a la comparación o contraste de estas fotos, como fue en este caso, los alumnos encuentran en este tipo de tareas una oportunidad para interpretar y expresar sus ideas libre y espontáneamente, un hecho que le permite al evaluador recolectar una interesante cantidad de información que luego puede utilizar para su análisis e interpretación de los datos. 
Para completar la tarea, se les pidió a los alumnos que, individualmente, observen, comparen y contrasten las dos fotos. Mientras los estudiantes grababan sus producciones, el docente, en su rol de evaluador, los escuchó sin interrumpirlos. Al mismo tiempo, tomó nota en las hojas de desempeño (Apéndice A) diseñadas especialmente para comentar y calificar la producción de cada alumno. En estas hojas, el docente detalló las áreas fonético-fonológicas y discursivas que los alumnos deberían modificar para mejorar su pronunciación. Finalmente, asignó a cada alumno una calificación. La evidencia escrita de esta información sirvió a dos propósitos: por un lado, como retroalimentación (en inglés, feedback) para que los alumnos identifiquen y clasifiquen sus errores, y puedan corregirlos de forma autónoma, y por el otro, como evidencia concreta de la cual se extrajeron las calificaciones que a cada alumno se le asignó por su desempeño.

Luego de haber grabado la tarea, los alumnos transcribieron ortográficamente ${ }^{3}$ sus producciones y resaltaron los aspectos fonético-fonológicos y discursivos que consideraban que debían ser mejorados. Posteriormente, se les solicitó que utilizaran la rúbrica de evaluación de trabajos orales (ver Cuadro 1), la cual había sido consensuada con ellos al inicio del curso, para auto-evaluar sus muestras de habla. Las buenas evaluaciones demandan la enunciación y explicitación de antemano de los criterios a utilizarse para determinar el nivel de la producción (Font Ribas, 2003; Litwin, 1998).

En el Cuadro 1 se detallan los aspectos de pronunciación y discursivos que conformaron la rúbrica para la asignación de puntajes -junto con los porcentajes asignados a cada elemento- que se tuvieron en cuenta para realizar las evaluaciones tantos de los alumnos como del docente.

Cuadro. 1. Rúbrica para la asignación de calificaciones de trabajos orales.

\begin{tabular}{llll}
\hline \multirow{2}{*}{$\begin{array}{l}\text { Elementos } \\
\text { de Pronunciación }\end{array}$} & Sonidos & $30 \%$ & $60 \%$ \\
\cline { 2 - 3 } & Acento, ritmo y entonación & $30 \%$ & \\
\hline \multirow{3}{*}{$\begin{array}{l}\text { Elementos } \\
\text { del Discurso Oral }\end{array}$} & $\begin{array}{l}\text { Planificación y Organización del discurso } \\
\text { oral }\end{array}$ & $10 \%$ & \multirow{2}{*}{$40 \%$} \\
\cline { 2 - 3 } & Inteligibilidad & $10 \%$ & \\
\cline { 2 - 3 } & Comprensibilidad & $5 \%$ & \\
\cline { 2 - 3 } & Gramática & $5 \%$ & $100 \%$ \\
\hline Promedio Total & & $50 \%$ & \\
\hline
\end{tabular}

Los alumnos realizaron las transcripciones de sus grabaciones utilizando el alfabeto ortográfico. Solo en aquellos casos en que los alumnos identificaron desviaciones fonológicas en sus producciones, a nivel segmental, por ejemplo, recurrieron a los símbolos fonéticos del IPA (International Phonetic Alphabet: https://www.internationalphoneticassociation.org/). Para las desviaciones a nivel suprasegmental, utilizaron las siguientes convenciones: (i) // para delimitar las fronteras de las frases entonativas, (ii) maYÚSsculas para indicar la presencia de acento o prominencia en cada sílaba afectada, (iii) subrayado de sílaba para resaltar la localizaCIÓN del acento nuclear (o sílaba tónica) y (iv) flechas delante de sílaba tónica para indicar la orientación del pitch (F0 frecuencia fundamental), por ejemplo: $\searrow$ indicaría que se trata de un tono descendente. 
Por su parte, el docente escuchó a cada alumno individualmente, tomó nota de información valiosa para ser devuelta luego en forma de feedback y, posteriormente, les asignó una calificación. Luego de que los alumnos completaron las tareas de evaluación y autoevaluación, el docente entregó a cada uno su hoja de desempeño con comentarios y su calificación. Los alumnos analizaron y compararon su autoevaluación con la evaluación realizada por el docente. Esto incluyó también la comparación de las calificaciones. En los casos en los que hubo discrepancia entre las calificaciones, el docente convocó al alumno en cuestión a una entrevista personal en la que ambos negociaron la evaluación para consensuar una calificación final.

Los autoinformes tuvieron un formato semi-estructurado, es decir, los alumnos fueron guiados por una serie de preguntas que debían contestar e ilustrar con ejemplos extraídos de sus producciones. En la primera pregunta, se les solicitó que describan brevemente los efectos de la implementación de la técnica de autoevaluación en el desarrollo de su pronunciación en LE. En la segunda, se les pidió que expliquen sucintamente los efectos que la tarea de evaluación negociada pudo haber tenido en la adquisición de una pronunciación adecuada en LE, y en la tercera pregunta se les sugirió que brinden opiniones y comentarios acerca de las dos tareas realizadas. Asimismo, se les pidió que, de ser posible, en cada una de sus respuestas, hicieran referencia a la toma de conciencia fonético-fonológica, la autonomía en el aprendizaje y a la motivación para continuar mejorando sus producciones orales.

\section{Resultados}

\subsection{Las calificaciones}

En el Cuadro 2 se muestran los resultados de la tarea de evaluación llevada a cabo por el docente y de autoevaluación realizada por los alumnos. Cabe aclarar en este punto que estos datos fueron registrados antes de la instancia de evaluación negociada entre los alumnos y el docente. La primera columna incluye la escala empleada para la asignación del puntaje (de Excelente a Insuficiente), mientras que en la segunda y tercera se presentan los resultados de la evaluación de los estudiantes y el docente, reflejados en número de alumnos (No. de alumnos) y en la media obtenida para los dos casos.

Cuadro. 2. Calificaciones de alumnos y docente.

\begin{tabular}{lllll}
\hline Conceptos & \multicolumn{2}{l}{ Calificación alumnos } & \multicolumn{2}{l}{ Calificación docente } \\
\hline & $\begin{array}{l}\text { No. de } \\
\text { alumnos }\end{array}$ & Media & No. de alumnos & Media \\
Excelente (10-9) & 0 & 0 & 0 & 0 \\
Muy Bien (9-8) & 0 & 0 & 3 & $17.54 \%$ \\
Bien (7-6) & 8 & $47.05 \%$ & 11 & $64.70 \%$ \\
Pobre (5-4) & 4 & $23.52 \%$ & 1 & $5.88 \%$ \\
Insuficiente (3-0) & 5 & $29.41 \%$ & 2 & $11.76 \%$ \\
\hline
\end{tabular}


Desde la perspectiva autoevaluativa de los alumnos, se observa que el $70.57 \%$ de los alumnos alcanzó los objetivos propuestos para la tarea. Los puntajes que se auto asignaron fueron distribuidos entre los conceptos de Bien, con un $47.05 \%$ y Pobre, alcanzando un $23.52 \%$. Por su parte, el docente asignó calificaciones más altas y a mayor cantidad de alumnos en comparación con los resultados de los estudiantes. Según el docente, el $88.12 \%$ de los alumnos obtuvo calificaciones por encima del concepto de Insuficiente, y la distribución de los conceptos comprende Muy bien, con un $17.54 \%$ hasta Pobre, con un 5.8\%. Es importante destacar que la cantidad de alumnos que consideró su desempeño como Insuficiente en esta tarea autoevaluativa (24.41\%) superó en un $17.65 \%$ al número de alumnos desaprobados por el docente $(11.76 \%)$. Esto indica que los alumnos parecieron ser más rigurosos con la asignación de sus calificaciones que su propio docente. Esta discusión será retomada oportunamente en la próxima sección.

Cabe destacar también que las instancias de negociación de las evaluaciones entre los alumnos y el docente se realizaron en un aula o en el mismo laboratorio de idiomas, y en forma personalizada con cada uno. Cada sesión de negociación duró aproximadamente entre tres o cuatro minutos y en ellas se discutieron algunas cuestiones relacionadas con el desempeño -aciertos y áreas por mejorar- de cada alumno. En solo un caso, una alumna mostró cierto grado de discrepancia con la calificación asignada por el docente y la suya. Asimismo, manifestó un alto grado de disconformidad con la implementación y el objetivo de las tareas de evaluación.

\subsection{Autoinformes evaluativos de los alumnos}

Una vez finalizada la actividad de evaluación negociada, se les pidió a los alumnos que en unas pocas líneas manifiesten sus impresiones y valoraciones acerca del empleo de dicha técnica en la clase de pronunciación inglesa, y que incluyan su opinión en cuanto a su incidencia, en caso de haberla, en sus procesos de aprendizaje (Apéndice B).

Sólo 1 de los 17 alumnos manifestó no estar de acuerdo con la implementación del trabajo conjunto para la evaluación de su producción (ver Alumno F), el resto destacó de un modo u otro su contribución para el reconocimiento de los errores; 5 estudiantes expresaron sentir inseguridad al autoevaluarse debido a no estar familiarizados con la técnica; 9 estudiantes hicieron referencia a la importancia de la negociación de la calificación con el docente, ya que ese encuentro les había ayudado a comprender mejor lo que se esperaba de ellos y los motivaba a continuar trabajando para mejorar su pronunciación en LE; 6 revelaron estar en desacuerdo con hacer transcripciones debido al tiempo que insumían pero, al mismo tiempo, reconocieron interesante el hecho de identificar sus propios errores y pensar o buscar posibles correcciones. Las siguientes son algunas de las reflexiones brindadas por distintos estudiantes al respecto:

Alumno A: Cuando transcribí mi presentación, y me escuché y tomé mayor conciencia de los errores que había cometido. Antes no reconocía, por ejemplo, las inflexiones de los tiempos verbales del pasado "visit - visitED”. Ahora, después de la grabación, creo que no me lo voy a olvidar más. Por supuesto, debo seguir practicando porque este es un proceso sin fin. 
Alumno B: A través de esta actividad me he dado cuenta del tipo de errores que cometo, y he podido corregir muchos de ellos por mi propia cuenta. Por ejemplo, antes acentuaba mal las palabras. Por ejemplo, "anaLYZE" la acentuaba en la última sílaba, como en español. Ahora, con las grabaciones y las correcciones del profesor me di cuenta de que se acentúa en la primera "ANAlize". Depende mucho de mí salir adelante y mejorar mi pronunciación, y con esto veo que se puede lograr. Este tipo de ejercicios me da seguridad y confianza en mí mismo.

Alumno C: Luego de hacer esta actividad, me di cuenta de los cambios que debo efectuar para lograr una buena pronunciación en inglés. No marcaba la sílaba tónica en las frases entonativas, ni diferenciaba los tonos. Ahora aprendí que puedo grabarme en casa, escucharme, transcribir mi discurso y así mejorar mi pronunciación. Me gusta esta estrategia.

Alumno D: Me gustó evaluar mi propio discurso. Sentí que yo era el docente. Adquirí muchas herramientas para mejorar mi pronunciación y entonación en el futuro.

Alumno E: Estaba convencido de no haber cometido tantos errores... sin embargo... aparecen en la grabación... Está bueno porque transcribir lo que dije me llevó a "ver" todo y ahí reconocí errores... Algunas frases no me sonaban correctas y como debía corregirme busqué en diccionarios y pregunté a un compañero que la tiene muy clara...

Alumno F: Lo que no me gustó fue cuando tuvimos que ponernos una nota y evaluar nuestro propio trabajo, porque es el docente quien debería evaluar, explicar los puntos débiles y mostrárselos al alumno, para luego intentar mejorar juntos.

Alumno G: En ocasiones necesitaríamos más feedback (cuando hay problemas serios) para poder mejorar. Cuando analizamos nuestro discurso y nos pusimos una nota, y después la comparamos con la tuya, me sentí bien. Había sido muy duro conmigo mismo, pero tu nota fue buena. Así que me hizo sentir mejor. Fue bueno para mi autoestima (¡Gracias!). Ahora quiero seguir aprendiendo. ¡Tal vez esto también sea bueno para otros alumnos frustrados!

En la próxima sección se discutirán algunas cuestiones relacionadas con el análisis e interpretación de los datos obtenidos, y se hará referencia a la hipótesis formulada junto con las tres preguntas planteadas, conducentes a la investigación.

\section{Discusión}

Las diferencias obtenidas en las calificaciones que presenta el Cuadro 2 abren un abanico de posibles conjeturas y cuestionamientos. ¿Acaso los alumnos eligieron calificaciones bajas por temor a desafiar en algún punto la figura de autoridad representada por el docente? Spencer-Oatey (2000) expresa que los alumnos, en ciertas circunstancias, pueden bajar sus propias notas guiados por el afán de mostrarse respetuosos y de reconocer las jerarquías en el aula, en un intento por evitar ser descor- 
teses con el docente. ¿Quizás se asignaron notas más bajas como resultado de identificar y tomar conciencia de las diferencias entre las formas fonético-fonológicas de su interlengua y el input al que son expuestos en sus clases de pronunciación? ¿Pudo la rúbrica empleada para la asignación de calificaciones de trabajos orales (Cuadro 1) generar dudas entre los alumnos al momento de ser utilizada? ¿Sería válido considerar que una posible falta de motivación o una auténtica baja autoimagen interfirieron en la decisión final al momento de autoevaluarse?

En el caso de que factores psicológicos hayan permeado sus propias calificaciones, recibir una valoración superior a la que ellos mismos se asignaron, debería afianzar sus autoesquemas. Usuga y Mesa (2016) expresan que los autoesquemas son sistemas de creencias que todo sujeto tiene sobre su persona y que afectan su funcionamiento y comportamiento en la vida. Estos comprenden tres aspectos: el autoconcepto, la autoestima y la autoeficacia y, sin lugar a duda, cada uno de ellos tiene incidencia en la motivación que puedan sentir -en este caso- los alumnos por superarse y seguir aprendiendo. Los resultados del Cuadro 2 sugieren la necesidad de rever, por ejemplo, los mensajes que los alumnos reciben en cuanto a sus cualidades y actos. Si los autoesquemas se construyen al interactuar con el medio, el obtener resultados positivos, debería retroalimentar la conducta del estudiante, de tal manera que se sienta competente, más autónomo y motivado para trabajar y superarse.

Previo a iniciar el análisis de los autoinformes es relevante aclarar un par de cuestiones. Por una parte, los autoinformes citados en la sección anterior fueron seleccionados al azar con excepción del realizado por el estudiante que se manifestó en disconformidad con esta dinámica de trabajo (Alumno F). Por otra parte, solo algunas de las contribuciones de los estudiantes fueron incluidas con el ánimo de satisfacer un doble propósito, el de ejemplificar la naturaleza de las reflexiones recopiladas y, a su vez, revelar cómo estas condujeron a las percepciones, deducciones y/o conclusiones que se detallan a continuación.

Las tres preguntas planteadas en este estudio fueron las siguientes:

1. ¿En qué medida la autoevaluación y la evaluación negociada contribuyen a despertar y estimular la concientización lingüística en los alumnos acerca del rol que cumple la pronunciación en el desarrollo del discurso oral en LE?

En algunos casos, los estudiantes resaltaron aspectos netamente discursivos como, por ejemplo, el incorrecto uso de la gramática y vocabulario o la falta de organización y lógica en sus ideas (Alumno E). También, señalaron algunas cuestiones relacionadas con los aspectos segmentales de la cadena discursiva, como la omisión de las inflexiones verbales en los tiempos del pasado: "visit"-"visitED" (Alumno A), o algunas desviaciones fonológicas en la realización de algunas consonantes, por ejemplo: /v/ vs. /b/. En otros casos, destacaron aspectos prosódicos, como por ejemplo la incorrecta localización del acento léxico en "anaLIZE" vs. "ANAlize" (Alumno B) y del acento nuclear dentro del marco de la frase entonativa, todo esto sumado a la incorrecta orientación de algunos tonos (Alumno C). Estos datos ejemplifican el rol que cumple la autoevaluación en tanto que funciona como un procedimiento de introspección que permite identificar y juzgar la propia conducta y pensamiento de los alumnos, con el objetivo de establecer las correcciones necesarias en su comportamiento. Esta técnica acciona como un disparador de la toma de conciencia de los alumnos para que mejoren su pronunciación en LE. 
2. ¿En qué medida la autoevaluación y la evaluación negociada favorecen el desarrollo de la autonomía en el aprendizaje?

Loubet (2007) entiende que las autoevaluaciones pueden ser pensadas como elementos coadyuvantes, permitiendo que el estudiante se perciba como participante autónomo y responsable de su propio proceso de aprendizaje, innovando así sus prácticas académicas. Fueron 16 los alumnos que admitieron que todo el procedimiento constituyó un método eficaz para auto-monitorear sus propias producciones, y así tomar mayor conciencia lingüística de la importancia de adquirir una pronunciación del inglés que les permita convertirse en hablantes eficaces $y$, al mismo tiempo, buenos docente y modelos de sus futuros alumnos y de otros hablantes de L2. Lograr un aprendizaje autónomo implica que los alumnos sean capaces de tomar decisiones en el aprendizaje, asumir compromiso en sus tareas, desarrollar un nivel de tolerancia a la frustración y una necesidad de logro que les motive a plantearse nuevos retos en el aprendizaje. Varias de estas cuestiones están plasmadas en los relatos de sus autoinformes: "A través de esta actividad me he dado cuenta del tipo de errores que cometo, y he podido corregir muchos de ellos por mi propia cuenta" (Alumno B); "....algunas frases no me sonaban correctas y como debía corregirme busqué en diccionarios y pregunté a un compañero que la tiene muy clara..." (Alumno E).

3. ¿En qué medida la autoevaluación y la evaluación negociada incentivan la motivación por mejorar el desarrollo de la pronunciación en LE?

Los autoinformes aportaron comentarios que permitieron establecer nexos entre la autoevaluación y la motivación de los estudiantes por mejorar su pronunciación en LE. Asimismo, la instancia de evaluación negociada, obtuvo un saldo positivo al resultar explícitamente mencionada y valorada por 9 de los participantes consultados. Los siguientes comentarios ilustran lo expuesto: “... este tipo de ejercicios me da seguridad y confianza en mí mismo" (Alumno B); "Ahora aprendí que puedo grabarme en casa, escucharme, transcribir mi discurso y así mejorar mi pronunciación... Me gusta esta estrategia..." (Alumno C). La reflexión de este último alumno claramente refleja la motivación que generó en él la técnica empleada para la autoevaluación. Otros estudiantes se manifestaron entusiastas tras evaluar su propia producción (Alumno D) o al compararla y discutirla con el docente (Alumno G).

En efecto, los comentarios de los estudiantes permitieron vislumbrar que la actividad de la autoevaluación y la experiencia de negociar la valoración con el docente, de un modo u otro, impactaron en su motivación, al punto de proyectar seguir trabajando en tareas futuras (Alumnos A, D) en pos de mejorar sus habilidades de pronunciación en la clase de inglés.

El análisis minucioso de estos datos revela que la hipótesis inicial se comprueba, porque la implementación de la autoevaluación y la evaluación negociada ofrecen una plétora de ventajas. En primer término, favorecen el aumento de la conciencia a nivel léxico, sintáctico, fonológico y discursivo, lo que -en las palabras de uno de los alumnos citados anteriormente-constituye uno de los pasos fundamentales hacia la adquisición de una L2. En segundo término, al permitir que los alumnos tomen decisiones reales y compartan con el docente la responsabilidad de evaluar sus trabajos, se los ubica en una posición de autocontrol dentro del entorno áulico, y esto es positivo para ellos 
dado que esta dinámica de trabajo los invita a ser participantes activos y más autónomos en su proceso de aprendizaje.

\section{Conclusión}

En este estudio se exploraron los efectos del empleo de la autoevaluación y evaluación negociada con relación al posible aumento en la toma de conciencia lingüística, la autonomía y la motivación en los alumnos. Según los hallazgos encontrados, la hipótesis inicial, en la que se mencionan los beneficios de incorporar ambas técnicas evaluativas en la clase de pronunciación del inglés como LE, es comprobada. Algunas consideraciones interesantes surgieron a raíz de los resultados de este trabajo basado en la idea de que, al incitar a los alumnos a tomar una postura activa de autorregulación ante el aprendizaje se gesta una motivación positiva en ellos que favorece el aprendizaje. Esta premisa se origina en la aseveración que Rogers hiciese en 1961, al advertir que "el único aprendizaje que realmente incide en el comportamiento es el que uno descubre y asimila por sus propios medios" (Dörnyei, 2000:131). Por tal motivo, la evaluación debe procurar que sus participantes estén en condiciones de desarrollar cierto grado de autonomía, autoestima y auto-valía propia (Celman, 1998; Koosha, Abdollahi \& Karimi, 2016). A raíz de los hallazgos encontrados en este trabajo, la próxima sección presentará una serie de limitaciones y se propondrá la realización de nuevas investigaciones en el área para corroborar lo aquí presentado y ampliar el estudio de este campo disciplinar.

\section{Limitaciones del estudio}

A pesar de que se pudo brindar respuesta a cada uno de los interrogantes planteados en este estudio, es oportuno delinear una serie de limitaciones. En primer lugar, no se pretende realizar generalizaciones de ningún tipo con relación a los hallazgos debido a que se trata de un estudio de corte interpretativo, en el que las creencias y opiniones de los alumnos - de carácter subjetivo- fueron el principal insumo de datos del estudio y por lo tanto no se puede hablar de mediciones. Para ello, hubiera sido necesario -y muy interesante por cierto- haber explorado la desviación fonológica de los alumnos mediante la utilización de ventanas acústicas. El análisis fonético hubiera permitido computar y dar cuenta de desviaciones en cuanto a intensidad de la emisión, duración sonora y altura tonal en cada muestra, por ejemplo. Queda entonces abierta la propuesta para retomar la investigación y evaluar cuantitativamente estos mismos datos y compararlos con los hallados aquí.

Por otro lado, el tamaño de la muestra poblacional fue reducido, es decir, solo 17 alumnos participaron en el trabajo. Una mayor muestra hubiera permitido ampliar el abanico de opiniones y creencias, aumentando de esta forma, la confiabilidad de los resultados. Otra cuestión es que la técnica objeto de estudio fue aplicada en un curso que tiene una duración de 4 meses y los datos fueron recopilados al realizar dos tareas específicas y de corta duración. A futuro sería interesante ampliar esta investigación y realizarla en otros contextos y con otras poblaciones de alumnos para comprobar si es posible avalar la efectividad de este tipo de tareas que tienen como 
objetivo lograr una mejor adquisición lingüística. Poner en acción esta dinámica de trabajo implicó una serie de pasos: escuchar a cada alumno en forma particular mientras grababan sus intervenciones, tomar nota de su desempeño, otorgarles una valoración global considerando la rúbrica para la asignación de calificaciones de trabajos orales (ver Cuadro 1) y, finalmente, negociar esa valoración con ellos. En efecto, todo el proceso demandó tiempo y esfuerzo por parte del docente. No obstante, al considerar el impacto que la actividad tuvo en el desarrollo de las habilidades de autoevaluación de los alumnos, así como en su autonomía de aprendizaje y su motivación para continuar perfeccionando su pronunciación, puede decirse que todo este arduo trabajo y el tiempo empleado bien valieron la pena.

\section{Referencias bibliográficas}

Altamimi, Ali. (2015): Improving English pronunciation among Arabic EFL school-age students using minimal pairs, [en línea]. Tesis de Maestría en Science in Education Teaching English to Speakers of Other Languages. Department of Language, Learning \& Leadership, State University of New York, at Fredonia Fredonia, New York. https://dspace.sunyconnect.suny.edu/bitstream/handle/1951/65691/Altamimi_Ali_Masters_Spring2015. pdf? sequence $=1$ [Consulta: 10 agosto 2018].

Bachman, Lyle. (2004): Statistical Analysis for Language Assessment, Cambridge, Cambridge University Press.

Baker-Smemoe, Wendy, y Naomi Haslam (2012): "The effect of language learning aptitude, strategy use and learning context on L2 pronunciation learning", Applied Linguistics, 34, 435-456.

Boud, David. (2003): Enhancing learning through self-assessment, Reino Unido, Routledge.

Boud, David, y James Kilty (2003): "Using self-assessment schedules in negotiated learning", en Boud, D., Enhancing learning through self-assessment, Reino Unido: Routledge, 102-108.

Celman, Susana. (1998): “¿Es posible mejorar la evaluación y trasformarla en herramienta de conocimiento?", en La evaluación de los aprendizajes en el debate didáctico contemporáneo, Buenos Aires, Paidós,

Coombe, Christine, y Christine Canning (2002): "Using self-assessment in the classroom: Rationale and suggested techniques", en Karen's Linguistics Issues (February). http:// www3.telus.net/linguisticsissues/selfassess2.html [consulta: 12 marzo 2017].

Dockinson, Leslie. (1995): "Autonomy and Motivation a Literature Review", System, 23, 165-174.

Dörnyei, Zoltán, y Peter Skehan (2003): "Individual differences in second language learning”, en Doughty, C., y M. Long (eds.), Handbook of Second Language Learning Malden, Blackwell Publishing, 580-630.

Dörnyei, Zoltán. (1998): Motivation in second and foreign language learning. Language Teaching, 31, 117-135.

Dörnyei, Zoltán. (2001): Teaching and Researching Motivation. London: Pearson Education Limited.

Dörnyei, Zoltán. (2012): Motivation in language learning. Shanghai: Shanghai Foreign Language Education Press.

Dörnyei, Zoltán, Alastair Henry y Christine Muir (2016): Motivational currents in language learning: Frameworks for focused interventions, New York, Routledge. 
Earl, Lorna, y Paul Lemahieu (1997): "Rethinking Assessment and Accountability", en Hargreaves, A. (ed.), Rethinking educational change with heart and mind: 1997 ASCD yearbook, Alexandria (VA), Association for Supervision and Curriculum Development, 149-167.

Farrell, Thomas, y George Jacobs (2010): Essentials for successful language teaching, New York, Continuum.

Fernández López, María Sonsoles (2017): Evaluación y aprendizaje. MarcoELE: Revista de Didáctica Español Lengua Extranjera, 24, 1-43.

Font Ribas, Antoni (2003): "Una experiencia de autoevaluación y evaluación negociada en un contexto de aprendizaje basado en problemas (ABP)", Revista de la Red Estatal de Docencia Universitaria, Universitat Politécnica de Valéncia, 3(2), 100-112.

Galaczi, Evenlina, Brechtje Post, Aike Li, Fiona Barker y Elaine Schmidt (2017): “Assessing second language pronunciation: Distinguishing features of rhythm in learner speech at different proficiency levels", en Isaacs, T., y P. Trofimovich, Second Language Pronunciation Assessment, Bristol, Multilingual Matters, 157-182.

Gardner, Robert (1988): “The Socio-educational Model of Second-language Learning: Assumptions, Findings, and Issues", Language Learning, 38, 101-126.

Isaac, Talía. (2014): “Assessing pronunciation”, en Aij, K. (ed.), The Companion to Language Assessment, Hoboken (NJ), John Wiley, 140-155.

Isaac, Talía, y Pavel Trofimovich (2017): "Key themes, constructs and interdisciplinary perspectives in second language pronunciation assessment", en Isaac, T., y P. Trofimovich, Second Language Pronunciation Assessment, Bristol, Blue Ridge Summit / Multilingual Matters, pp. 3-11.

Isaac, Talia, Pavel Trofimovich, Guoxing Yu y Bernardita Chereu (2015): "Examining the linguistic aspects of speech that most efficiently discriminate between upper levels of the revised IELTS pronunciation scale", IELTS Research Report Online, 4(1), 1-48.

Jenkins, Jennifer (2000): The phonology of English as an International Language, Oxford, Oxford University Press.

Johnson, David, y Roger Johnson (2004): Assessing students in groups. Promoting group responsibility and individual accountability, Estados Unidos, Corwin Press. Inc.

Koosha, Mansur, Abdollahi Azam y Karini Fatemeh (2016): “The Relationship among EFL Learners' Self-Esteem, Autonomy and Reading Comprehension", Theory and Practice in Language Studies, 6(1), 68-78.

Krashen, Stephen (1985): The input hypothesis, New York, Longman.

Levis, John (2005): "Changing contexts and shifting paradigms in pronunciation teaching", TESOL Quarterly, 39, 369-377.

Litwin, Edith (1998): "La evaluación: campo de controversias o un nuevo lugar para una buena enseñanza", en Camillioni, A., S. Cellman, E. Litwin y M. Palou de Mate, La evaluación de los aprendizajes en el debate didáctico contemporáneo, Buenos Aires, Paidós, 11-35.

Loubet, Roxana (2007). "Procesos de evaluación formativa y el desarrollo de la autonomía y responsabilidad en estudiantes universitarios" [en línea], en Centro de Investigaciones y Servicios Educativos (CISE), Universidad Autónoma de Sinaloa, México. https://www. researchgate.net/publication/228605181_Procesos_de_evaluacion_formativa_y_el_desarrollo_de_la_autonomia_y_responsabilidad_en_estudiantes_universitarios [Consulta: 3 agosto 2018].

Murphy, Tim. (1998): "Stages of facilitation: Conceptualizing learner development”, Nanzan University's LT Briefs, 8, 26-27. 
Murphy, John, Amanda Baker (2015): "History of ESL pronunciation teaching", en Reed, M., y L. John, The handbook of English pronunciation, Malasia, Wiley Blackwell, 36-67.

Morley, Joan (1991): "The pronunciation component in teaching English to speakers of other languages", TESOL Quarterly, 25(3), 481-520.

Morley, Joan (1994): Pronunciation pedagogy and theory: New views, new dimensions. Alexandria (VA), TESOL.

Moyer, Alene (2014): "Exceptional outcomes in L2 phonology: The critical factors of learners engagement and self-regulation", Applied Linguistics, 35(4), 418-440.

Moyer, Alene (2015): “Autonomy in Second Language Phonology: Choice vs. Limits", en Language Teaching, Cambridge, Cambridge University Press, 1-17.

Munro, Murray, y Tracey Derwing (1995): "Foreign accent, comprehensibility and intelligibility in the speech of second language learners", Language Learning, 45, 73-97.

Munro, Murray, y Tracey Derwing (1998): "The effect of speaking rate on listener evaluations of native and foreign-accented speech", Language Learning, 48, 159-182.

Munro, Murray, y Tracey Derwing. (2015): "Intelligibility in research and practice: Teaching priorities", en Reed, M., y J. Levis, The Handbook of English Pronunciation, West Sussex, Wiley Blackwell, 377-396.

Ordorica, Daniel (2010): "Motivación de los alumnos universitarios para estudiar inglés como lengua extranjera", en LeAA Lenguas en Aprendizaje Autodirigido. Revista Electrónica de la Mediateca del CELE-UNAM. México, UNAM / CELE, 3(2).

Oscarson, Mats (1989): "Self-assessment of language proficiency: Rationale and applications", Language Testing, 6, 1-13.

Petchprasert, Anongnad. (2012): "Feedback in second language teaching and learning", US-China Foreign Language, 10(4), 1112-1120.

Rose, Heath (2012): "Reconceptualizing strategic learning in the face of self-regulation: Throwing language learning strategies out with the bathwater", Applied Linguistics, 33(1), 92-98.

Schmidt, Richard (2001): “Attention”, en ROBINSON, P. (ed.), Cognition and second language instruction, Cambridge, Cambridge University Press, 3-32.

Singhanuwananon, Supatranut (2016): "Intelligibility redefinition and students' confidence in English speaking in Thai ELT", European Journal of Sustainable Development. 5(4), 209-215.

Spencer-Oatey, Helen (2000): "Rapport management: A framework for analysis”, en Spencer-Oatey, H. (ed.). Culturally speaking: Managing rapport through talk across cultures, London, Open Linguistics Series, Continuum, 11-46.

Taylor, David (1991): "Who speaks English to whom? The question of teaching English", System, 19(4), 425-435.

Tomlin, Russell, y Víctor Villa (1994): "Attention in Cognitive Science and Second Language Acquisition", Studies in Second Language Acquisition, 16, 183-203.

Underhill, Nic (1987): Testing Spoken Language, Cambridge, Cambridge University Press.

Ushioda, Ema (2001): "Language learning at university: Exploring the role of motivational thinking”, en Dörnyei, Z., y R. Schmidt (eds.), Motivation and Second Language Acquisition, Honolulu (HI) University of Hawaii Press, 99-125.

Usuga, Alison, y Sara Mesa (2016): "La influencia de factores psicológicos como autoestima, autoconcepto y autoeficiencia en el rendimiento académico en adolescentes", Psyconex: Psicología, psicoanálisis y conexiones. Departamento de Psicología, Universidad de Antioquia. Medellín, Colombia, 8(13), 1-11.

Won, My (2015): “A qualitative examination of teacher-student power-sharing in Chinese 
classrooms: A study in Hong Kong", Frontiers of Education in China Journal, 10(2), 251-273.

Yates, Lynda, Beth Zielinski, y Elizabeth Pryor (2011): "The assessment of pronunciation and the new IELTS pronunciation scale", en OSBORNE, J. (ed.), IELTS Research Reports, 12, Melborne / Mancherster, IDP IELTS Australia and British Council, 1-46.

\section{Apéndices}

\section{Apéndice A. Hoja de desempeño y devolución (Feedback sheet)}

$\begin{array}{ll}\text { Universidad Nacional de Mar del Plata } & \text { Name: } \ldots \ldots \ldots \ldots \ldots \ldots \ldots \ldots \ldots \ldots \\ \begin{array}{l}\text { Profesorado de Inglés } \\ \text { Discurso Oral II }\end{array} & \text { Date: } \ldots \ldots \ldots \ldots \ldots \ldots \ldots \ldots \ldots \ldots\end{array}$

\section{Pronunciation}

\section{FEEDBACK SHEET}

- Sounds

- Prosody

Other oral discourse features

- Planning and organization of discourse

- Intelligibility (understanding)

- Comprehensibility (ease of understanding)

- Use of grammar and vocabulary

\begin{tabular}{|c|c|}
\hline Strengths & Weaknesses \\
\hline & \\
& \\
\hline
\end{tabular}

FINAL GRADE:

\section{Apéndice B. Autoinformes evaluativos}

Universidad Nacional de Mar del Plata Profesorado de Inglés

Discurso Oral II
Name:

Date:

\section{SELF ASSESSMENT REPORT}

(Note: For questions $1 \& 2$ below, please make sure in your answers you refer to awareness raising, learner autonomy and motivation to keep on learning pronunciation.) 
1. Briefly describe the effects on your pronunciation development of having implemented self evaluation (illustrate your claims with examples from your recording).

2. Briefly describe the effects on your pronunciation development of having implemented negotiated evaluation in the pronunciation class (illustrate your claims with examples from your recording).

3. Other comments. 